\title{
Tips to Assemble the Berkeley Lab Cosmic Ray Detector
}

\section{Colleen Twitty, Fred Bieser and Howard Matis}

Lawrence Berkeley National Laboratory

June 2000 (Version 1.01)

\section{Int roduction}

The Berkeley Comic ray consists of several items. Cosmic rays strike two scintillator paddles and produce a few photons of lights. These photons are detected by a phototube. A phototube is glued to each of the scintillator paddles. A PC board provides power for the phototube, processes the signals, and displays the number of cosmic rays. A CBL interf ace can be used to digitize the data. A wooden box provides mechanical support and protection for the detector. Appendix I lists an abbreviated list of the components needed to build the detector.

The following information is a guide to assembling the detector. For more information on this project, please contact:

How ard Matis

MS 70-319

Law rence Berkeley National Laboratory

Berkeley, CA 94720

Alternatively, you can send e-mail to HSMatis@lbl.gov.

Simple cosmic ray detectors previously have been made at the Stanford Linear Accelerator Center (SLAC. More details of the SLAC detector can be found in SLAC publication ${ }^{1}$ SLAC-TN-95-1.

A student at Berkeley Lab, Henry Cheng, prepared an earlier version of this report. There is an excellent description of machining the scintillator and gluing it to a phototube. This can be found on the Web at:

http:// csee.lbl.gov/cup/Su98/HCheng/homepage.htm

${ }^{1}$ Eric Harpell, Willy Langeveld, Don McShurley, Steve Shapiro, John Venuti, The CCRT: an inexpensive comic ray muon detector. Willy Langeveld is the main contact. 


\section{Assembling the box}

The box consists of seven wood pieces and one Lucite piece. Appendix $D$ contains a detailed description and shows the dimensions of the box.

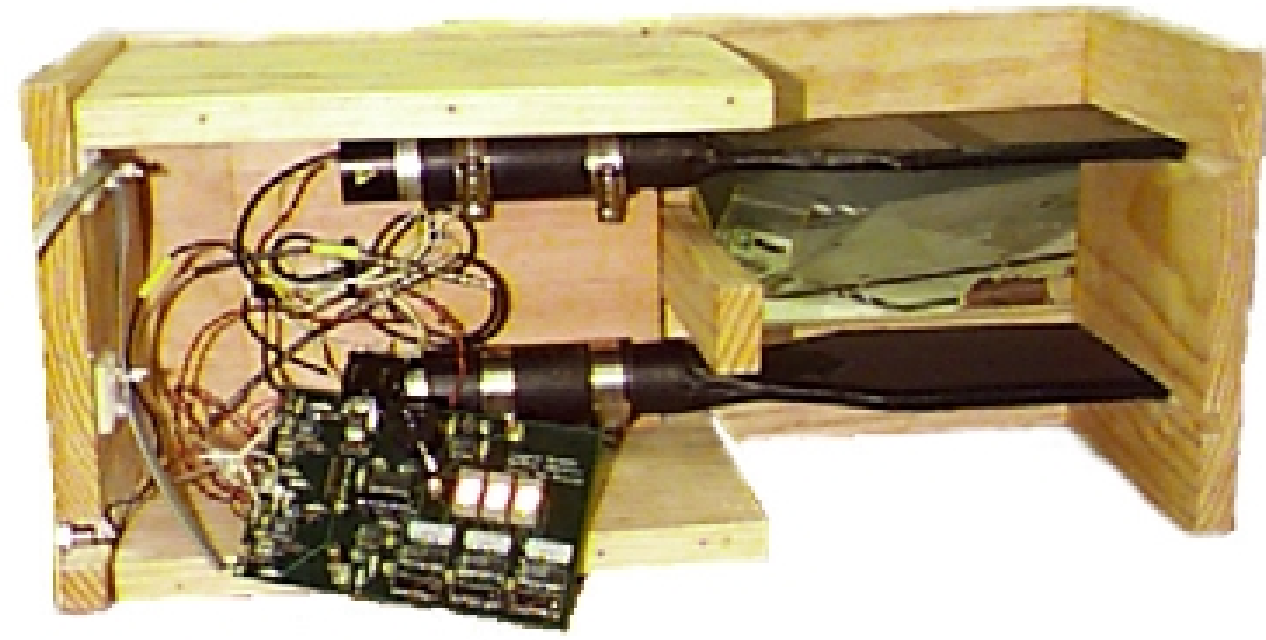

The wood pieces are attached using wood screws and a screwdriver. Afterwards, you should sand and stain the box to protect its surface. The Lucite piece is not attached until after the coincidence circuit has been assembled and attached to it.

Installing the power connector

The wood is too thick to mount the power connector, so you need to replace a portion of the box with a small aluminum plate, which can support the connector. Drill a hole for the connector in an aluminum plate. Drill two holes through the plate for screws, one on either side of the hole for the connector. Remove a piece of the box at the top edge (which meets the Lucite) of the piece behind the ends of the phototubes. The hole must be large enough for the connector, but small enough for the plate to be screwed on over it. Screw the plate over the hole. Attach the connector. Solder a wire from the square hole of "DC in" on the circuit board (power) to the center pin of the connector. Solder the wire from the round hole (ground) to the outside pin.

CBL

Drill a hole large enough for the CBL cord. Keep in mind where you plan to put the screws to hold the Lucite.

\section{Making the scintillator}

The scintillator must be glued to the phototube so that that a minimum of light is lost at the interface of the tube and scintillator. It is best if one end of the scintillator is less than 
or equal to the diameter of the face of the phototube so that the light traveling through the narrow end of the scintillator strikes the phototube. You want the other end of the scintillator to be wider so that the detector can count more cosmic rays. The phototube must be able to support the weight of the scintillator when it is glued. Also, keep in mind the size

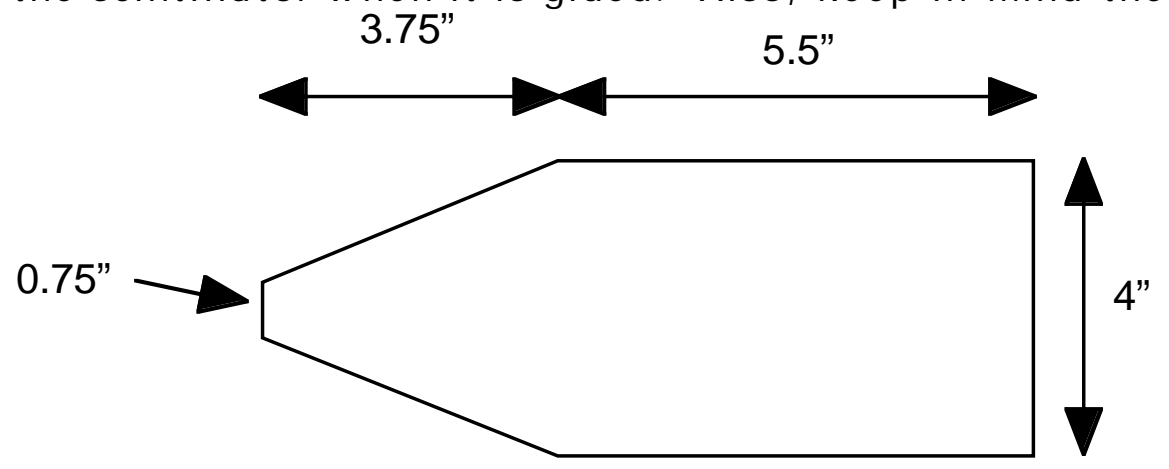

box you plan to use to hold the paddles. The phototubes that we used had a diameter of 3/4". We used the following dimensions for the scintillators:

Polishing the scintillator

After the scintillators are cut into desired shape and size, polish all sides of the scintillator, except the top and bottom surface.

First, polish all sides of the scintillator using 600-grid sandpaper. Before polishing, wet the sandpaper first. Wash off all dirt and dust occasionally while sanding the scintillator.

Then use a rotating round-table machine designed for polishing plastic and scintillators to do a second polishing. Cover the rotating table with Putz Pomade, a polishing material that is closed to 800 grid. Turn on the machine and hold on to scintillator while the machine is polishing the scintillator. Let the machine polish the scintillator for 30 seconds for each side. Then turn the scintillator around and polish for another 30 seconds for each side. Wash off all Putz Pomade on the scintillator.

Then use the same machine, but this time cover it with alumina powder. Sprinkle the powder over the round-table and pour a bit warm water to make it some sort of paste. This paste is equivalent to 1200 grid. Use the same technique of polishing as described above. Wash the scintillator. Always wash scintillators with cold or room temperature water.

Gluing the scintillator to the phototube After the scintillator is done being polished, it is ready to be glued to the phototube. We use gravity to make sure the entire surface of the scintillator is glued evenly onto the phototube. We use a stand to keep the phototube from falling over (but the 
stand never actually touches the phototube). We put the scintillator on a flat surface to make sure it is 90 degrees upright. Them we clip it to some heavy solid to maintain its position.

We use a glue that is a mixture of "826" and "U". The mixture is 3 to 1 respectively. We first have to warm up the both liquid separately and the mix it with the above recipe. Do not mix too much of it because we only need a little. Then vacuum it with an air pump so that there are no air bubbles in the mixture. Then it is ready to glue the scintillator to the phototube.

Use easy-to-remove tape to cover the area that is close to the tip of the scintillator so that the glue won't stick to the surface of the scintillator. Apply the glue on the tip surface of the scintillator and put the phototube on top of it. Then peel off the tape by the tip of the scintillator. Wait at least 3 hours for the glue to solidify. Do not touch this glue with your fingers!

Wrapping

Before gluing the scintillator to the tube, cut two pieces of black cardboard for the top and bottom surfaces of the scintillator. The size of the cardboard should be a tiny bit smaller than the surface of the scintillator. After the scintillator is glued to the tube, it is ready to be wrapped.

First, wrap the scintillator with aluminum foil. Avoid contact with the phototube because the end of the tube will be at high voltage. Then tape the two already-cut cardboard pieces onto the top and bottom surfaces of the scintillator. Make sure that the cardboard is touching the glass of the phototube. Then use black tape to cover all sides of the scintillator. Also, use the black tape to wrap around the contact area between the phototube and the scintillator.

\section{Assembling the PC board}

We have created a PC Board, which holds the electronics for the detector. You will need the following items to assemble the PC Board.

1. damp sponge

2. clean soldering iron

3. soldering iron

4. solder

5. solder wick (to remove solder) 
The soldering iron can be dangerous. Make sure that you are careful when working with it. It can produce very severe burns. Make sure it rests in a secure area so that it does not cause a fire.

Select a clean well-lit place to work. You should choose a comfortable chair at an appropriate height.

Put the resistors on the board.

The resistors are banded for identification. The identification scheme can be found in Appendix B. To check that you are working with the correct pieces, compare the part name with the band colors listed on the parts list. The fourth band is gold on every resistor. By convention, each resistor on the board is positioned so that each gold band lies in the same direction. For example, you could put all the bands on the right for the horizontal resistors and down for the vertical resistors.

Pick a resistor to start with and consult the Parts List, which will tell you the corresponding location on the board. The resistor itself should lie on the labeled side of the board, with its wires extending to the other side.

To place a resistor onto the board, gently bend the two pieces of wire in the same direction close to the resistor. The resistor should be roughly perpendicular to the bent wire, making a square U-shape. Slide the wires into the designated holes in on the board until the resistor is touching the board. Hold the resistor on the board, turn the board over, and bend the two wires away from each other slightly. They need to be bent so that they can remain on the board when it is turned upsidedown.

Solder the resistors onto the board.

Place the board face down on your workspace. Using a soldering iron and solder, attach each resistor to the board. Solder on the backside of the board. Touch the tip of the soldering iron to the wire of the resistor and the metallic pad on the board. Hold the soldering iron in one hand and the solder in the other. Unwind some of the solder so that you have a piece extended from your hand. Touch this piece to the hot pad and wire of the resistor while keeping the iron in place. Heat makes the solder flow. Feed a small amount of solder, then remove the soldering wire, keeping the soldering iron in place for a few seconds. Finally, remove the soldering iron. You do not want to take a long time soldering the parts because the high temperatures will eventually damage them. The solder should completely cover the pad, and should stick to the wire and the pad. If it does not, try using the soldering iron to apply more heat. 
Check your soldering joints; they should completely cover the metallic pad and surround the wire. Then use wire cutters to cut off the wire just above the mound of solder.

Place the capacitors on the board.

Choose a capacitor and slide it into the corresponding location. Remember to bend the wires to hold the capacitor on the board. Some capacitors are marked with a "+" or "-" sign. For these capacitors, there is a corresponding "+" or "-" marked on the board. Put the " + " on the capacitor on the same side of the board as the " + " on the board. The same should be done when the capacitor and board are both marked with "-" signs. If the board and capacitor give no indication of charge, the placement of the capacitor does not matter.

Solder the capacitors onto the board.

Once you have stuffed the board with the capacitors, solder these joints. Then cut the excess wire.

Place the integrated circuits on the board.

Each of these pieces has many pins that can make them tricky to put on the board. If a piece does not stay firmly on the board, use your fingernail to bend one of the legs to keep it in place.

Solder the integrated circuits.

These pieces have many pins and it is important the solder makes a good connection between each pin and the board.

Put the remaining pieces on the board and solder. Diodes: Place the diodes on the board like resistors, but use the following orientation: On the board, each box indicating the position of the diode is split into two boxes, a larger one and a smaller one. The thick black band on one end of the diode should be placed above the smaller box.

Buzzer: Place the buzzer on the board, making sure to put the pin on the " + " side of the buzzer in the square hole.

Switches: Make sure the switches are flush with the board before soldering. You may need to use tape to hold them in place.

LED: This piece can melt easily with heat. Therefore, do not push this piece all the way down onto the board. Instead, leave a few millimeters between the base of the piece and the board. Solder the wires well, but do it as quickly as possible. The longer leg must go in the square hole. 
Regulator and Heat Sink: The regulator, at U1, needs a heat sink. The heat sink must be lower than the top of the LED numbers, so trim the heat sink if needed.

\section{Connect the CBL}

Cut back 1/2" to 1 " of the gray plastic CBL cord without cutting the wires inside. Strip the white and black wires, by approximately $1 / 3$ of an inch. This should be long enough to solder onto the board. Make sure that you have most of the wire rather than a few strands. If you accidentally cut too much of the wire, strip more, cutting back more of the gray covering. Try to make both of the wires approximately the same length.

Twist the wires so that they will slide into the hole more easily. Pre-tin the wires. Put the black wire (ground) through the round $\mathrm{CBL}$ hole, and the white wire (signal) through the square CBL hole. Solder each wire to the board, and trim the excess. The CBL cord plugs into the "DIG IN" channel on the CBL. To test the CBL, you need to use the appropriate programs, and you must connect your TI calculator to the CBL with the cord that comes with you calculator.

\section{At $t$ ach the power supply wires}

Power is connected to DCin. The square hole is for power; the round one is ground. Use a 12 -volt power source. Once the box is built, a power source-the transformer or power supply that can be used with your car-will attach to a connector. The board will receive power from wires, which are soldered from this connector to DCin. In the meantime, you can test the board by using any 12 -volt source.

\section{Connect the photomultiplier tube wires}

First, strip the yellow and white wires of the phototube. ${ }^{2}$ Twist the ends of the two wires together and solder them. Wrap the end of the wires in tape. Do the same with the other phototube.

Strip the thin red and black wires, exposing approximately $1 / 4$ $1 / 3$ inch, so that they can be soldered to the board. Try to remove just the plastic, leaving as much wire as possible. If you have cut through most of the wire, start over by stripping

\footnotetext{
${ }^{2}$ These instructions are for the model P30CW5 phototube manufactured by Electron Tubes Limited, 100 Forge Way, Unit F, Rockaway, NJ 07866, USA. They can be reached at 800-521-8382 or at phototubes@aol.com. Other manufacturer's tubes can be used. However, these instructions might need to be modified.
} 
again. Try to get each wire approximately the same length. Twist each wire, so that it will more readily slide onto the board. Pre-tin the wire by applying a little solder and heat before trying to attach it to the board. Slide the black wire (ground) into the round hole of DClin, and slide the red wire (power) into the square hole. Solder each wire to the board, trimming the excess. The signal wire goes into the square Siglin. The ground wire goes in the round hole.

Follow the same procedure with the other phototube, using DC2In and Sig2In.

\section{If wires break}

The wires supplying power, carrying signal to the phototubes, and connecting to the CBL are all prone to breaking at the point where they are soldered on. If this happens, remove the wire from the board and clear the hole. Re-strip and re-solder the wire. Once the board is completed and mounted on the Lucite, which, in turn, is screwed onto the box, the wires should cease detaching because they should not be experiencing any more strain. Until then, handle the board gently, and avoid pulling on the wires.

\section{Strain relief}

Install the strain relief just below the hole for the CBL wire. The strain relief absorbs the stress of someone pulling on the wire instead of allowing the wire to pull on the board. Use plastic ties, if necessary, to hold the wire firmly. Install a second strain relief for added support. Before securing the wire to the strain relief, check that you are leaving an appropriate amount of slack. Test the strain relief by pulling on the wire a few times.

\section{Adjusting the phototubes}

Turn the detector on and have it count singles on the phototube you are adjusting. Using a small, narrow screwdriver, turn the screw in the back of the phototube until the count rate is hundreds per minute and is approximately the same on each phototube. To check that you have eliminated the background noise, remove the paddles from the detector. Keep them parallel to the ground, but separate them as much as possible. Have the detector count in coincidence. The count should be zero or a few counts. Check this several times. Then put the phototubes in the detectors, turn it on, and count in coincidence. You now are counting cosmic rays. 
You can increase the count rate by putting a radioactive source near the scintillator because the scintillator responds to the radiation.

\section{Installing the paddles into the box}

Hose clamps will secure the paddles in the box. To cushion the phototubes, they are wrapped in a 4" Iong strip of rubber. Trim the width of the rubber until it wraps around the phototube and the ends meet or come very close to meeting without overlapping. The ends should meet such that when the phototube is put into the box, the ends are not visible. The rubber should cover the phototubes, leaving approximately an inch and a half at the bottom to affix a high voltage label. Use small black pieces of tape to secure the rubber tightly around the phototube. These pieces of tape should not be visible after putting the phototubes into the box.

Punch a hole in each of the four hose clamps, remembering you need to adjust these clamps. Using a pencil and ruler, mark where you will put the screws to hold the clamps. This spot should be situated so that it will hold the scintillator parallel with the sides and in the middle of the groove in the box. Slide the paddles in the box to get a sense of where the clamps need to go to effectively support the phototubes, and stay over the rubber to protect them. Pre-drill the holes and attach the clamps. Put the phototubes in the clamps and tighten the clamps with a screwdriver.

Put a strain relief between the two phototubes. Use a large plastic tie to bundle the extra wire, looping the tie through the strain relief. Leave some slack for removing the circuit board or Lucite.

\section{Lucite}

The Lucite panel provides support for the PC board. It is mounted on the wooden box.

First drill holes for the screws into the box and countersink them appropriately. The PC Board has several switches, which are used to attach it to the Lucite panel. The switches require 1/4-inch holes and the buzzer needs a 1-inch hole. These holes do not need countersinks. Before drilling the holes, mark the locations with permanent marker. This can later be removed with alcohol.

\section{Acknowledgements}

This project was supported by a generous grant from the William F. and Edith R. Meggers Project Award from the 
American Institute of Physics. Additional support was provided by Department of Energy funding to Nuclear Science Division and the Center for Science and Engineering at the Lawrence Berkeley National Laboratory. . Additionally, we would like to thank Electron Tubes, which is located in Rockaway New Jersey. They very graciously donated half of the phototubes that we produced. Their support enabled us to produce twice as many complete detectors. We thank Henry Cheng for writing part of the section on machining the scintillator. 


\section{Appendix A: List of Materials}

1. assembling the PC board:

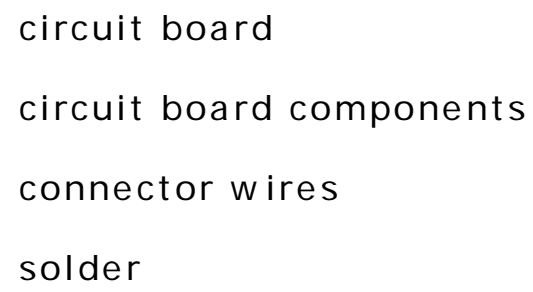

2. building the wooden box:

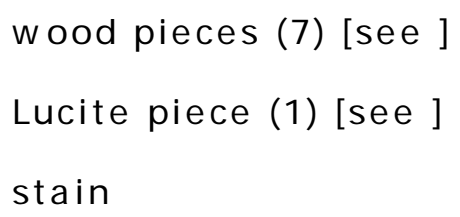

3. mounting the scintillator paddles:

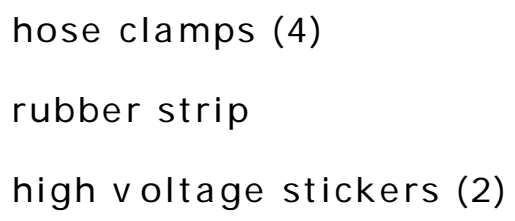

4. making the paddles:

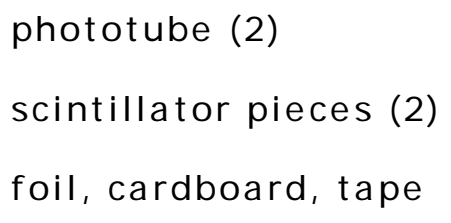

5. wood screws:

6. mounting the connector:

7. aluminum plate

8. connector (chassis, compatible with transformer)

9. 12-volt transformer 


\section{Appendix B: Resistor Color Codes}

Each resistor is labeled by three colored bands. These bands identify the resistance of the resistor. The first two bands indicate the first two digits of the resistance while the third band indicates the multiplier of 10 . The units are ohms. The colors are identified by:
0 black
1 brown
2 red
3 orange
4 yellow
5 green
6 blue
7 violet
8 gray
9 white

For instance, suppose you find a resistor that is coded blue-redorange. The numerical values are from the above table-6-2-3. The resistance is 60 (the first number is the tens digit) plus 2 for times 10 raised to the third power. Therefore, the resistance of this resistor can be calculated as

$$
(60+2) \cdot 10^{3}=62,000 \Omega
$$

Often, there is a fourth band. A gold band indicates that the accuracy of the resistor is $1 \%$; a silver band shows that it is $5 \%$. If there is no fourth band, then the tolerance is $20 \%$ 


\section{Appendix C: Parts List}

Resistors:

Quantity 3

2

3

4

4

4

2

1
3

1

1

\section{Capacitors:}

Quantity 2

2

14

1

1

1

\section{Int egrated circuits:}

\begin{tabular}{ll} 
Quantity & Location \\
\cline { 1 - 2 } 1 & U1 \\
2 & U16, U2 \\
2 & U3, U12 \\
1 & U4 \\
1 & U5
\end{tabular}
C21

C16

C13

C4
Part and Band Colors $1.1 \mathrm{k} \Omega$ : brown, brown, red

$1.1 \mathrm{k} \Omega$ : brown, brow $\mathrm{n}$, red

$51 \Omega$ : green, brown, black $10 \mathrm{k} \Omega$ : brown, black, orange $11 \mathrm{k} \Omega$ : brown, brown, orange $12 \mathrm{k} \Omega$ : brown, red, orange $100 \mathrm{k} \Omega$ : brown, black, y ellow

$100 \mathrm{k} \Omega$ : (variable)

$4.7 \mathrm{k} \Omega$ : yellow, violet, red

$470 \Omega$ : yellow, violet, brown $120 \mathrm{k} \Omega$ : brown, red, yellow

Part

$6.8 \mathrm{mf}$ $100 \mathrm{pf}$

$0.1 \mathrm{mf}$

C3, C6, C7, C8, C9, C17, C18, C19, C20,

$330 \mathrm{mf}$ $0.0022 \mathrm{mf}$ $1 \mathrm{mf}$

Part LM 7805CT

NE555 DS9696

7408

7400 


$\begin{array}{lll}3 & \text { U6, U9, U13 } & 7 \text { seg } \\ 3 & \text { U7, U10, U14 } & 74590 \\ 1 & \text { U8 } & 7447\end{array}$

Other Parts:

Quantity 2

1

Location

D1, D4

D2

D3

diode

buz z er

$\mathrm{S} 1, \mathrm{~S} 2, \mathrm{~S} 3$

led

JP1, JP2, JP3, JP4, JP5, Header 2 JP6 


\section{Appendix D: \\ Dimensions for the Box Assembly}

The box, which holds the electronics and scintillator, consists of seven pieces. The overall assembly diagram is show $n$ in below:

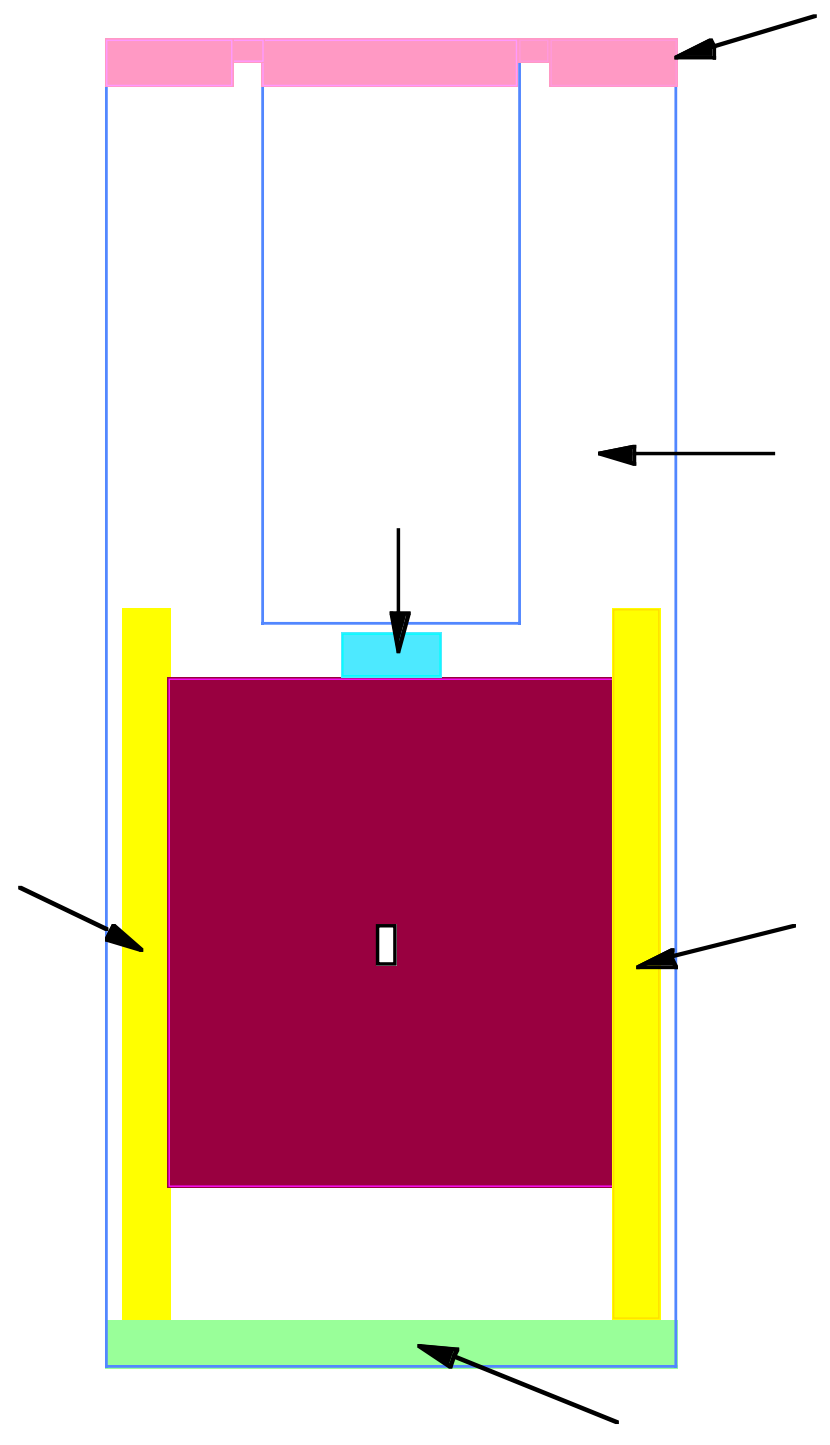


The following table shows the dimensions of the individual pieces of the box. The number refers to the previous diagram.

Rectangular Pieces

\begin{tabular}{|l|l|l|l|l|}
\hline Number & Quantity & Width (inch) & Height $($ Inch) & Thickness (inch) \\
\hline 1 & 1 & 6.5 & 9 & 0.75 \\
\hline 2 & 1 & 6.5 & 9 & 0.75 \\
\hline 3 & 2 & 6.5 & 11.25 & 0.75 \\
\hline 4 & 1 & 21 & 9 & 0.75 \\
\hline 5 & 1 & 7 & 8 & 1.5 \\
\hline 6 & 1 & 1.5 & 6.5 & 0.75 \\
\hline
\end{tabular}

Item \#1 has a cutout in its center. Its dimensions are described below: $21^{\prime \prime}$

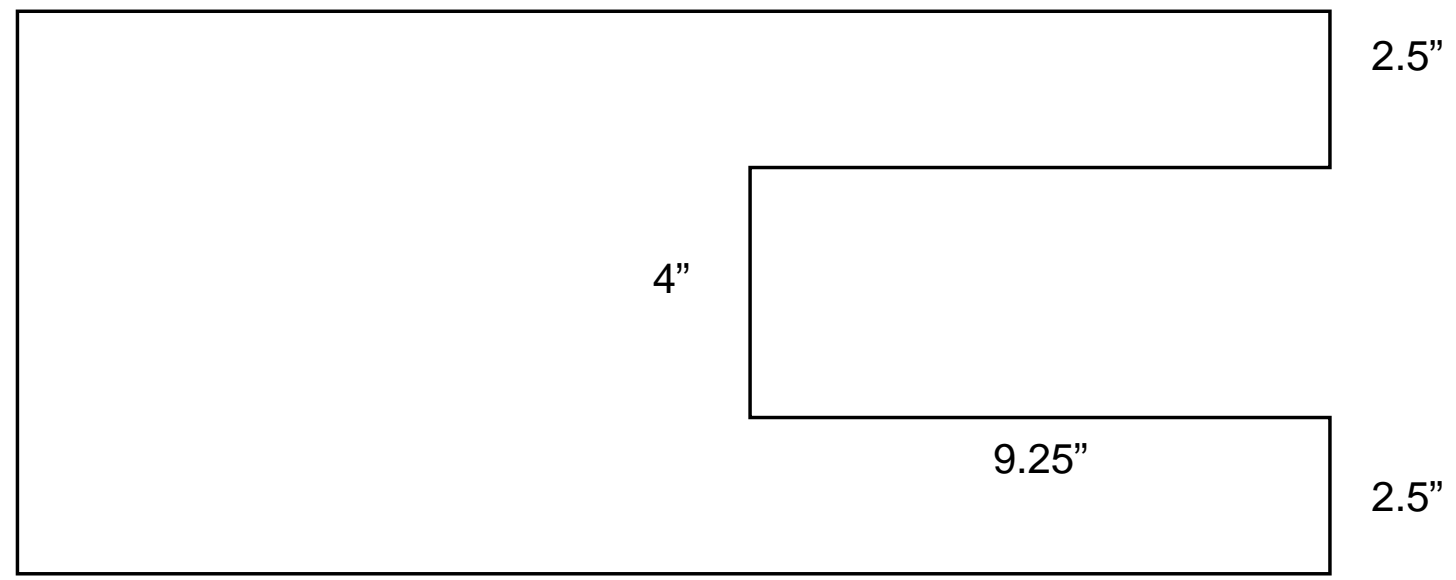

The top of the box is a Lucite piece, which is cut with the same dimensions of item 1 .

Items 2 need to be modified in the following way. First, Item \#2 has two groves. The following picture shows the location:

\begin{tabular}{|c|c|c|c|c|}
\hline 2" & $0.5 "$ & 4" & $0.5 "$ & 2" \\
\hline & ᄂ & & $\neg$ & \\
\hline
\end{tabular}

The depth of this grove is $0.375^{\prime \prime}$. 


\section{Appendix E: Circuit Diagram for PC Board}

The following diagram is a schematic for the PC board. 


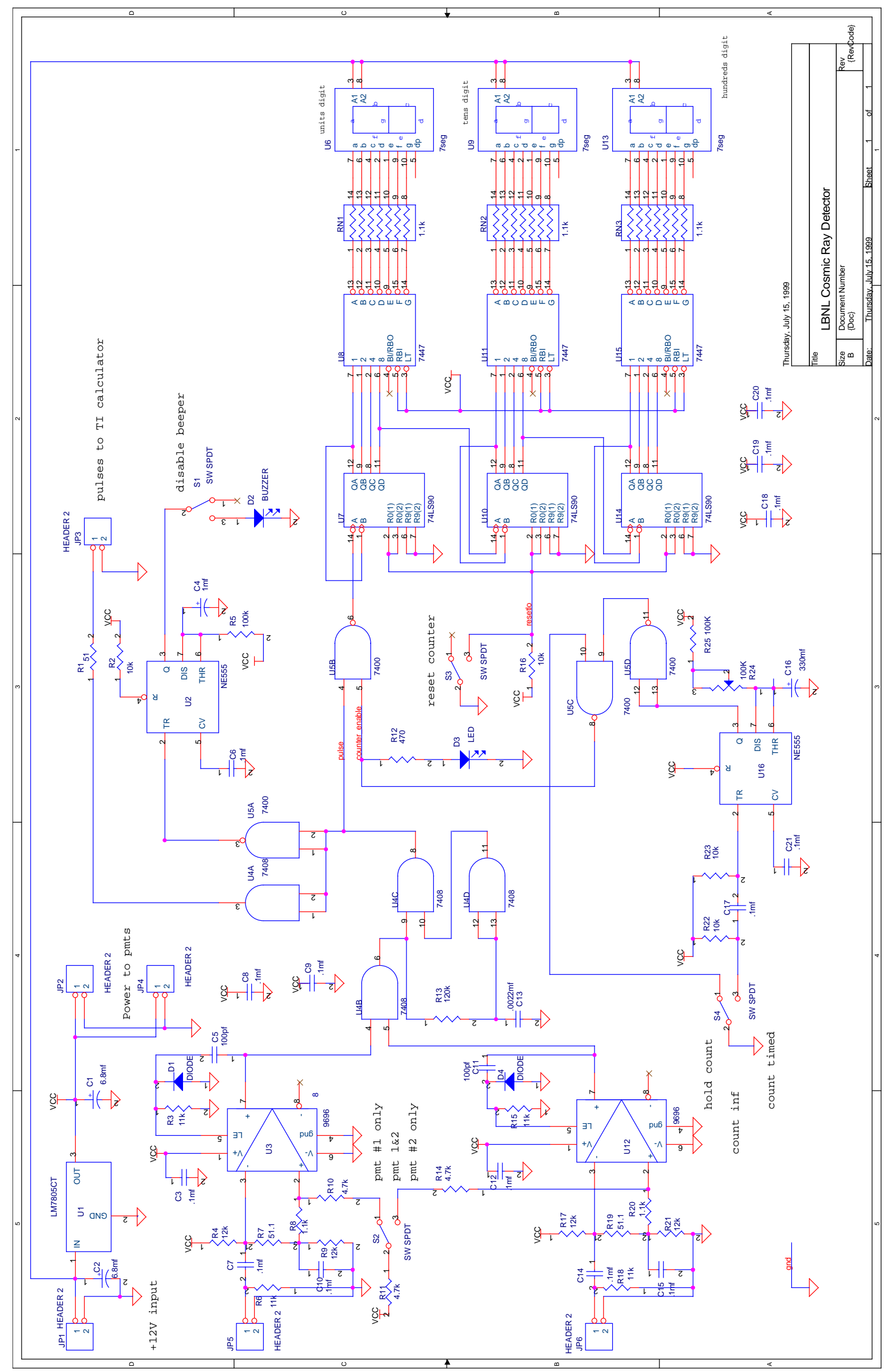

